\title{
COMPARISON OF HIPPARCOS RESULTS ON THE SAME GREAT CIRCLE SCANNED ON DIFFERENT DATES
}

\author{
HANS SCHRIJVER \\ SRON Space Research Utrecht \\ Sorbonnelaan 2 \\ 3584 CA Utrecht \\ The Netherlands
}

\begin{abstract}
The star abscissae as obtained from the reduction of Hipparcos observations have been compared with results obtained on the same circle, but on a different date. The comparison shows that the results are reproducible, taking into account the expected random and non-random effects.
\end{abstract}

\section{Principles and results}

The axis of the Hipparcos scanning motion, following the path prescribed by its scanning law (Perryman and Vaghi 1989), regularly comes back to an earlier position. In such cases, a great circle is scanned that is close to a previously scanned one. The scanning direction can be the same, or inverse. We have taken advantage of this fact by comparing abscissae determined from such pairs of scans, in order to evaluate the reproducibility of the results. Before comparison the abscissae have been corrected for all known astrometric effects: aberration caused by earth and spacecraft motion, proper motion, parallax, gravitational deflection.

Up to now three such pairs of observation sets, with opposite scanning directions, have been compared.

The observed differences show a random noise with r.m.s. of about 9 milli-arcseconds, and a systematic effect sinusoidal in the abscissa with an amplitude of 7-10 milli-arcseconds. The random part is due to statistical effects due to photon noise, inaccuracies in the Input Catalogue, and unknown proper motions and parallax distribution. It can be shown (Schrijver 1992) that its size agrees with expectations. The systematic effect corresponds in size and phase with the effect expected from the average unknown parallaxes.

Details can be found in Schrijver (1992).

\section{Acknowledgements}

The contribution to this result by colleagues from ESOC and the FAST data reduction consortium is gratefully acknowledged. The work in Utrecht has been supported by the Space Research Organization of the Netherlands, SRON.

\section{References}

Perryman, M.A.C., Vaghi, S., 1989, ESA SP-1111, Vol. I, 11

Schrijver, H., 1992, Astr. Astrophys., accepted 